\title{
Surgical Treatment for Primary Cardiac Leiomyosarcoma Causing Right Ventricular Outflow Obstruction
}

\author{
Kazunori Ishikawa, MD*; Shuichiro Takanashi, MD; Wahei Mihara, MD; \\ Toshihiro Fukui, MD; Yasuyuki Hosoda, MD
}

\begin{abstract}
A 55-year-old man was admitted to another hospital because of dry cough and dyspnea that rapidly worsened before admission. Chest computed tomography revealed a low-density mass that nearly obstructed the main pulmonary arterial trunk. Pulmonary thromboembolism was suspected and treated with catheter-directed thrombolysis therapy. Despite optimal thrombolysis and anticoagulant therapy, his symptoms persisted. He was referred for further examination and possible surgery for presumed pulmonary thromboembolism. The mass appeared more likely to be a tumor than a thrombus on careful analysis of the magnetic resonance imaging. At surgery, the anterior wall of the main pulmonary arterial trunk, the pulmonary valve, annulus, and the right ventricular outflow tract were all invaded by what was found to be a tumor and were resected under conventional cardiopulmonary bypass. The resected area was reconstructed with a $25-\mathrm{mm}$-diameter bioprosthetic valve and Xenomedica patch. Final pathological diagnosis was primary cardiac leiomyosarcoma involving the pulmonary valve. Postoperative course was uneventful, and he was discharged 11 days after surgery without adjuvant therapy because he refused it. Ten months later, the patient was well, but a chest X-ray revealed some coin lesions in the bilateral lung fields that were thought to be metastatic tumor. (Circ J 2005; 69: 121-123)
\end{abstract}

Key Words: Adjuvant therapy; Primary cardiac leiomyosarcoma; Thromboembolism

$\mathbf{P}$ rimary cardiac leiomyosarcoma is extremely rare, constituting less than $0.25 \%$ of all cardiac tumors, but it has a lethal prognosis even if surgical resection, with or without adjuvant therapy, is performed, 2,3 Only a few cases of surgical resection of cardiac leiomyosarcoma have been reported? We present a case of surgical resection of a primary cardiac leiomyosarcoma involving the pulmonary valve, which was reconstructed with a bioprosthetic valve and Xenomedica patch.

\section{Case Report}

A 55-year-man was admitted to another hospital because of dry cough and exertional breathlessness that had rapidly worsened over the previous 1-2 weeks. He had no remarkable medical history. A computed tomographic (CT) examination of the chest revealed a filling defect that almost completely obstructed the main pulmonary arterial trunk.

A diagnosis of pulmonary thromboembolism was assumed, and the patient was treated with catheter-directed thrombolysis therapy over 3 days. However, his dyspnea and shortness of breath worsened despite an optimal regimen of thrombolytic and anticoagulation therapy. The patient was referred to us for further evaluation and possible surgical management of the presumed pulmonary thromboembolism.

Upon admission, laboratory results showed elevated lactate dehydrogenase (785 U/L) and liver enzymes (AST

(Received September 16, 2003; revised manuscript received December 26, 2003; accepted January 20, 2004)

Department of Cardiovascular Surgery, Shin Tokyo Hospital, Matsudo and *Department of Cardiovascular Surgery, Hoshi General Hospital, Koriyama, Japan

Mailing address: Kazunori Ishikawa, MD, Department of Cardiovascular Surgery, Hoshi General Hospital, 2-1-16 Ohmachi, Koriyama, Fukushima 963-8501, Japan. E-mail: k-ishikawa@hoshipital.or.jp
49 U/L, ALT 95 U/L). Chest X-ray showed mild cardiomegaly with a cardiothoracic ratio of $52 \%$. The patient underwent magnetic resonance imaging (MRI) of the chest, which revealed a solid mass at the pulmonary valve, measuring approximately $50 \times 40 \times 30 \mathrm{~mm}$ with low signal intensity on T1-weighted image and increased signal intensity on $\mathrm{T}_{2}$-weighted image, that appeared to be invading the main pulmonary arterial trunk (Fig 1a,b). There was no evidence of distal thromboembolism in either of the pulmonary arteries. Because the mass appeared to be a tumor rather than a thrombus, the possibility of malignancy was raised. Transthoracic echocardiography disclosed a large mass involving the pulmonary valve and extending to the main pulmonary arterial trunk, enlargement of the right ventricle (end-diastolic diameter, $52 \mathrm{~mm}$ ), and displacement of the ventricular septum toward the left ventricle. The mass almost completely obstructed the main pulmonary arterial trunk (Fig 1c), and right ventricular failure was suspected. The patient was scheduled to undergo surgery for tumor extirpation and histological evaluation of the mass.

At surgery, a hard mass was palpated in the slightly dilated main pulmonary arterial trunk. Conventional cardiopulmonary bypass was established with ascending aortic perfusion and bicaval drainage through a median sternotomy. After the heart was arrested, the main pulmonary arterial trunk was incised longitudinally from just above the pulmonary valve to the bifurcation, whereupon a whitish tumor originating from the pulmonary valve was found to have almost completely blocked the lumen of the main pulmonary arterial trunk and invaded its anterior wall. We also opened the right pulmonary artery longitudinally behind the ascending aorta and recognized scattered tumor on the posterior wall of the right main pulmonary artery that extended far distally, at least to the hilum, such that radical resection appeared impossible. The mass was resected as completely as possible, together with the anterior wall of 
$\mathbf{a}$

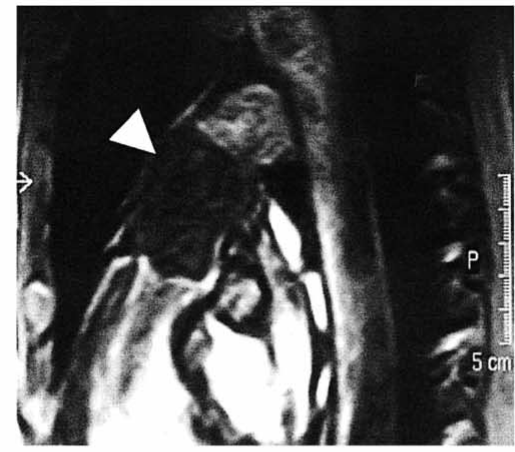

b

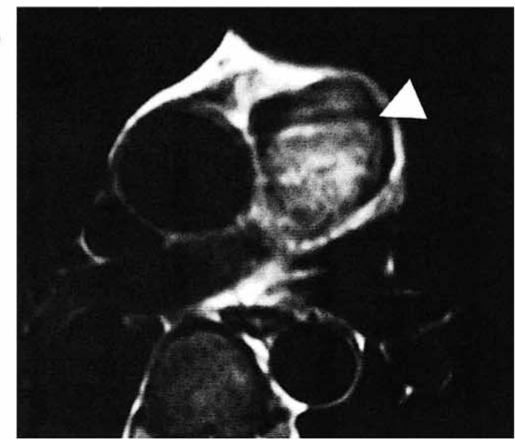

c

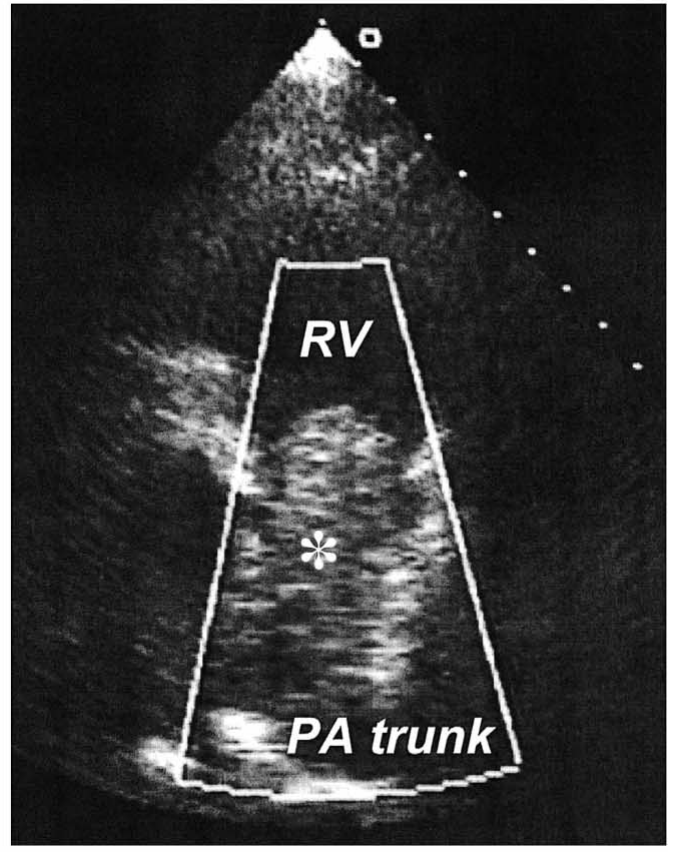

Fig 1. Preoperative magnetic resonance imaging reveals a huge tumor growing within the pulmonary arterial trunk and obstructing it nearly completely (arrow): (a) T1-weighted sagittal image, (b) T2-weighted transversal image). (c) Transthoracic echocardiogram shows a large mass $(*)$ in the pulmonary arterial trunk.

a

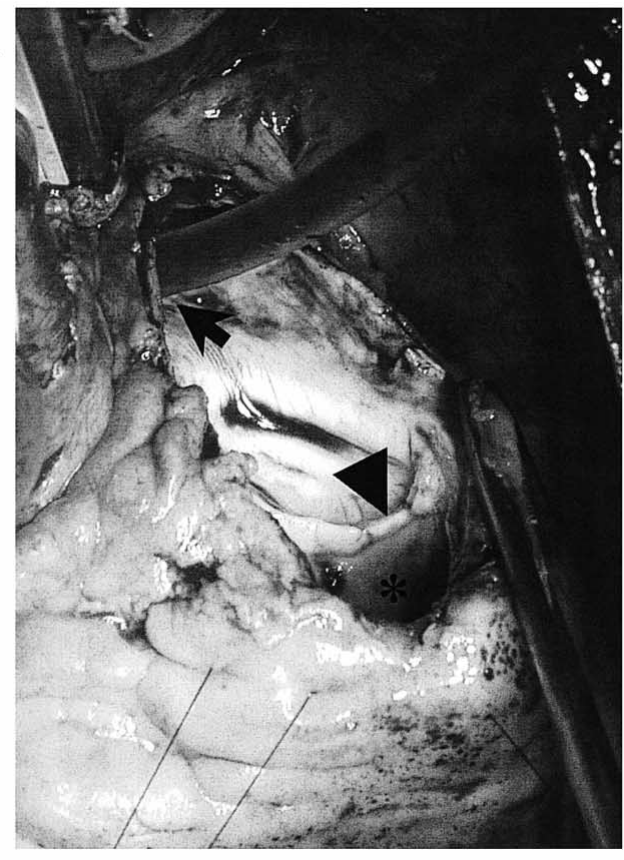

b

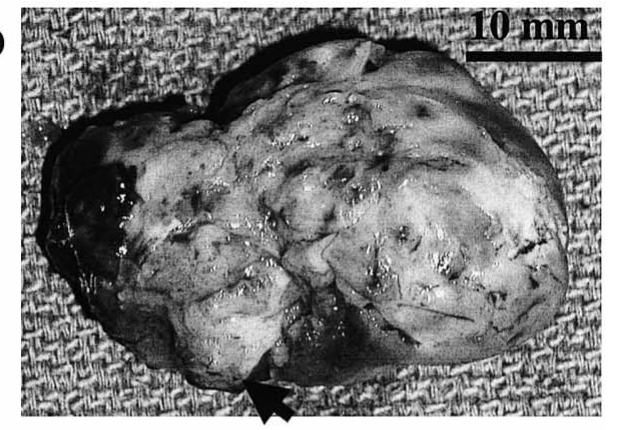

c

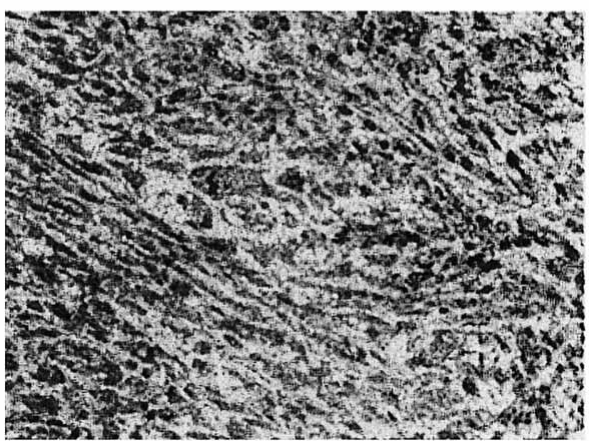

Fig 2. (a) Intraoperative view after resection of the anterior wall of the main pulmonary arterial trunk, the pulmonary valve, annulus (arrowhead), and the right ventricular outflow tract $(*)$, all of which were invaded by the tumor. Suction was inserted into the right main pulmonary artery (arrow). (b) Specimen of the resected tumor showing tumor adherent to the pulmonary valve annulus, and a remnant of the pulmonary valve (arrow). (c) Light microscopy shows spindle cells with a high rate of mitosis, indicating malignancy (hematoxylin and eosin stain, $\times 200$ ).

the main pulmonary arterial trunk, the pulmonary valve, annulus, and right ventricular outflow tract (Fig 2a,b). The resected area was reconstructed with a $25-\mathrm{mm}$ diameter bioprosthetic valve (Carpentier-Edwards PERIMOUNT bioprosthesis, Edwards-Lifesciences, Irvine, CA, USA) and Xenomedica patch (Baxter Healthcare Corp, Horw, Switzerland). After the procedure was completed, the patient was easily weaned from bypass.

Histopathological examination revealed spindle-type cells with a high rate of mitosis, indicating malignancy 
(Fig 2c). Immunohistochemical staining showed the tumor cells to be positive for vimentin, $\alpha$-smooth muscle cell actin, and myosin, but negative for desmin, striated muscle actin, and CD34, indicating that the tumor had smooth muscle cell origin. These findings suggested a leiomyogenic malignant tumor, and because of the absence of a primary lesion in other organs, a diagnosis of primary leiomyosarcoma of the heart was made.

The patient's postoperative course was uneventful, and he was discharged 11 days after surgery with neither adjuvant chemotherapy nor radiation therapy because he refused both. Two months after surgery, a chest X-ray revealed a coin lesion in his right middle lung field that was thought to be a metastatic tumor. To date, 10 months after surgery, some coin lesions have been found in the bilateral lung fields on his chest X-ray, but there has not been any evidence of recurrence of the tumor on his heart itself; he has been well with almost no limitations in his daily life.

\section{Discussion}

Primary cardiac neoplasms are rare; approximately $25 \%$ are malignant and almost all of them are sarcomal There are many types of sarcomas, including rhabdomyosarcoma, osteosarcoma, fibrosarcoma, angiosarcoma, liposarcoma, and leiomyosarcoma, but primary leiomyosarcoma of the heart is the rarest among them, constituting less than $0.25 \%$ of all cardiac tumors! In the literature only 54 cases have been reported since 19664

Diagnosis of primary cardiac leiomyosarcoma originating from the right ventricular outflow tract is very difficult because the clinical symptoms are usually similar to those of pulmonary thromboembolism, which makes not only early diagnosis difficult but also leads to misdiagnosis? Unfortunately, clinical presentation varies, from dyspnea on effort with or without, dry cough to chest pain and hemoptysis, which occur mostly only in the advanced stage of the disease ${ }^{1-5}$ Most previous cases have been diagnosed post mortem, but recent advances in diagnostic techniques such as echocardiography, CT, and MRI have made it possible to diagnose it in living patients. Several reports suggest that CT and MRI may be the most useful modalities for differentiation between tumor and thrombotic material. Therefore, the mass appeared to be a tumor rather than a thrombus and most likely malignant. In particular, heterogenous enhancement on MRI after application of gadolinium-diethylene-triamine-pentaacetic acid is characteristic of a vascularized tumor? The usefulness of biopsy via cardiac catheterization to make an accurate preoperative diagnosis has also been reported in the literature? In the present case, a solid mass presented with low signal intensity on $\mathrm{T}_{1}$-weighted image and increased signal intensity on T2-weighted image by MRI and there was no evidence of distal thromboembolism in either of the pulmonary arteries on CT or MRI examination.

The mean survival time of patients with leiomyosarcoma of the heart is 6 months after diagnosis, 10 and quality of life is poor because of progressive right heart dysfunction. ${ }^{-5,9,10}$ Because it originates from the heart itself, the tumor is not easy to resect completely. Despite apparently complete surgical excision, most patients die within the first year after diagnosis because of local tumor relapse or metastatic disease ${ }^{11,12}$ The aims of the surgical procedure are to relieve symptoms and to offer adequate palliation. In the present patient, because of the extensive distribution of the tumor in the posterior wall of the right main pulmonary artery, radical tumor resection was not possible. Extended resection followed by reconstruction with the bioprosthetic valve and Xenomedica patch was performed with the hope of relief of his symptoms.

The fact that some patients have survived more than 2 years after surgery with adjuvant therapy ${ }^{9,13}$ suggests that the combination of adjuvant therapy and excision of the tumor is somewhat effective, but the actual effectiveness and indications for such therapy are currently not clearly established because of the rarity of experience with leiomyosarcoma?

\section{Conclusion}

We report a surgical case of primary cardiac leiomyosarcoma involving the pulmonary valve. Ten months after resection of the tumor, the patient was well with almost no limitations in his daily life. There was no evidence of recurrence of the tumor on his heart itself, but a chest X-ray revealed some coin lesions in the bilateral lung fields that we presume to be metastatic tumor. Further careful followup of this patient is necessary.

\section{References}

1. Bruke AP, Cowan D, Virmani R. Primary sarcomas of the heart. Cancer 1992; 69: 387-395.

2. Mayer E, Kriegsmann J, Gaumann A, Kauczor HU, Dahm M, Hake U, et al. Surgical treatment of pulmonary artery sarcoma. J Throac Cardiovasc Surg 2001; 121: $77-82$.

3. Zerkowski HR, Hofmann HS, Gybels I, Knolle J. Primary sarcoma of pulmonary artery and valve: Multimodality chemotherapy and homograft replacement. J Thorac Cardiovasc Surg 1996; 112: 1122 1124.

4. Schroder S, Walker T, Greschniok A, Herdeg C, Karsch R, Ziemer G. Primary cardiac leiomyosarcoma originating from the pulmonary valve. J Cardiovasc Surg 2001; 42: 53-56.

5. Anderson MB, Kriett JM, Kaplanski DP, Tarazi R, Jamieson SW. Primary pulmonary artery sarcoma: A report of six cases. Ann Thorac Surg 1995; 59: 1487-1490.

6. Kauczor HU, Schwickert HC, Mayer E, Kersjes W, Moll R, Schweden F. Pulmonary artery sarcoma mimicking chronic thromboembolic disease: Computed tomography and magnetic resonance imaging findings. Cardiovasc Intervent Radiol 1994; 17: 185-189.

7. Rafal RB, Nichols JN, Markisz JA. Pulmonary artery sarcoma: Diagnosis and postoperative follow-up with gadolinium-diethylenetriamine-pentaacetic-acid enhanced magnetic resonance imaging. Mayo Clin Proc 1995; 70: 173-176.

8. Winchester PA, Khilnani NM, Trost DW, Litvak B, Gold JP, Sos TA. Endovascular catheter biopsy of a pulmonary artery sarcoma. Am J Roentgenol 1996; 167: 657-659.

9. Antunes MJ, Vanderdonck KM, Andrade CM, Rebelo LS. Primary cardiac leiomyosarcomas. Ann Thorac Surg 1991; 51: 999-1001.

10. Nguyen KT, Mak K, Sanfilippo AJ, Rosen WS,Cheeseman FD. Primary left atrial leiomyosarcoma simulating pulmonary thromboembolism. Can Assoc Radiol J 1994; 45: 48-51.

11. Thomas CR, Johnson GW, Stoddard MF, Clifford S. Primary malignant cardiac tumors: Update 1992. Med Pediatr Oncol 1992; 20: 519-531.

12. Llombart-Cussac A, Pivot X, Contesso G, Rhor-Alvrado A, Delord JP, Spielmann M, et al. Adjuvant chemotherapy for primary cardiac sarcomas: The IGR experience. Br J Cancer 1998; 78: 1624-1628.

13. Okabayashi H, Fujiwara Y, Kanzaki Y, Mitsudou K, Yamamoto H. A case report of primary leiomyosarcoma of the heart. Jpn J Thorac Surg 1987; 35: 2161-2165. 